\title{
A Theoretical Study of the Formation of Benzene Excimer: Effects of Geometry Relaxation and Spin-state Dependence
}

\author{
Dongwook Kim \\ Department of Chemistry, Kyonggi University, Suwon 443-760, Korea. E-mail: dongwook-kim@kyonggi.ac.kr \\ Received May 9, 2014, Accepted May 16, 2014

\begin{abstract}
Geometry relaxation effects on the formation of benzene excimer were investigated by means of $a b$ initio calculation at SOS-CIS( $\left.\mathrm{D}_{0}\right)$ /aug-cc-pVDZ level. In the case of T-shaped dimer configuration, intermolecular interactions in the excited states are found to be nearly the same as those in the ground state and structural deformations are limited within a single molecule; the geometry relaxation effects are then negligible and singlet-triplet energy gap remains constant. As for face-to-face eclipsed dimer, on the other hand, both molecules undergo structural change. As a result, intermolecular interactions in the excited states are significantly different than those in the ground state. Although the intermolecular distances obtained from potential energy curve calculation with frozen molecular structures are in qualitative agreement, the excitedstate binding energies are notably overestimated with respect to those at optimized structures. In particular, the effects are calculated to be larger in $T_{1}$ state and hence singlet-triplet energy gap, which reduces markedly in this configuration, is underestimated without relaxation.
\end{abstract}

Key Words : Benzene excimer, Geometry relaxation effects, Singlet vs triplet, Ab initio calculation

\section{Introduction}

Intermolecular interactions, in particular among the $\pi$ conjugated molecules, have been of long-lasting interest. They are known to play instrumental roles in various biological phenomena ${ }^{1-3}$ and supramolecular chemistry., ${ }^{4,5}$ Recently, the interactions are newly highlighted as organic photonics and electronics of next generation utilize $\pi$ conjugated materials ${ }^{6,7}$ and interactions among them can modify their intrinsic properties. ${ }^{8-11}$ While excited state phenomena in the solid-state organic semiconductors are involved in these devices, ${ }^{9,12-14}$ most of the studies thus far have focused on the interactions among the ground-state molecules. ${ }^{4,5,15}$ In this regard, intermolecular interactions in the excited states attract attention increasingly. $2,3,8,10,16-26$

As for recent theoretical studies of intermolecular interactions in the excited states, various acene molecules including benzene were employed. ${ }^{9,18,20-22,24-27}$ Most of those studies, however, rely on the frozen molecular structures derived from the ground-state calculations and thus the geometric relaxation effects have been neglected. In this regard, it is demanding to assess how much the structures are deformed in the excited state and investigate what impacts they have. In addition, studies thus far put more emphasis on the ability of theory to reproduce experimental values such as excimer binding energies, equilibrium intermolecular distances, the spectral shift with respect to the fluorescence of monomer, etc., which are associated with singlet excited states. However, triplet state dynamics also play an essential role in organic electronics, ${ }^{8-11}$ for example, triplet exciton is the major product in organic light emitting diodes (OLEDs) and triplet-triplet annihilation is believed to be one of the

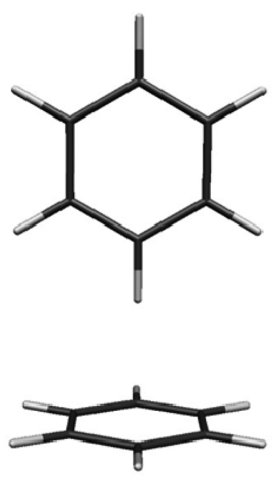

T-shaped
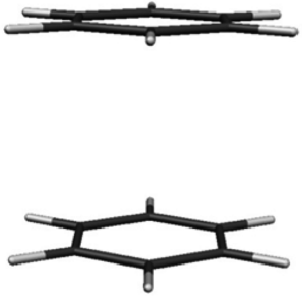

F2F-eclipsed
Figure 1. Molecular structures of representative benzene dimers: T-shaped (left) and face-to-face eclipsed (right) configurations.

main phenomena to influence OLED efficiency. ${ }^{27,28}$ Furthermore, the conversion between singlet and triplet states in large depends upon the energy difference of these states. ${ }^{13}$ Therefore, triplet excimers deserve as much attention as singlet ones.

In this report, we detail the results of $a b$ initio calculations on the formation of benzene excimer. T-shaped (T) and faceto-face eclipsed (F2F-E) configurations are taken into account (Figure 1); these configurations turn out to serve as representative examples for ones in which intermolecular interactions in the excited states are quite similar to and different than those in the ground states, respectively. We discuss the geometry relaxation effects of both benzene molecule and its dimer in the excited states and their dependence on spin state. 


\section{Computational Details}

Ground state geometries of both single molecule and dimer of benzene are optimized at scaled opposite spin (SOS)-MP ${ }^{29}$ level of theory using Dunning's aug-cc-pVDZ basis sets, and optimal geometries are also derived at SOSCIS $\left(D_{0}\right)^{30}$ level of theory for both the lowest singlet $\left(S_{1}\right)$ and triplet $\left(\mathrm{T}_{1}\right)$ states. To evaluate binding energies of benzene dimer in the excited states, potential energy curves are computed as a function of intermolecular spacing; hence, molecular structures remain fixed. In order to investigate the effect of geometry relaxation, dimer structures in the excited states are further optimized at SOS-CIS $\left(\mathrm{D}_{0}\right)$ level of theory. The size of basis set in this study is quite limited and thus the effect of its incompleteness is inevitable. To assess this effect, we computed basis set superposition error by the counterpoise method. ${ }^{31}$ To shed more light on the characteristics of excited states, natural transition orbital (NTO) analyses were also carried out using respective optimized geometry. All these calculations were conducted using Qchem package version $4.0 .^{32}$

\section{Results and Discussion}

Monomer: Frozen vs Relaxed Geometry. In order to better understand dimer in the excited states, we first investigated benzene molecule itself; the geometric features of benzene in both ground $\left(\mathrm{S}_{0}\right)$ and lowest excited $\left(\mathrm{S}_{1} / \mathrm{T}_{1}\right)$ states and its electronic transition energies were calculated and collected in Table 1 . With regard to geometric parameters, only $\mathrm{C}-\mathrm{C}$ and $\mathrm{C}-\mathrm{H}$ bond lengths are considered due to the high symmetry of benzene. When going from the ground state to the first excited states $\left(S_{1}\right.$ and $\left.T_{1}\right)$, the geometric deformations are found to mainly occur in $\mathrm{C}-\mathrm{C}$ bond while the $\mathrm{C}-\mathrm{H}$ bond lengths remain intact. Therefore, we will mainly focus on the $\mathrm{C}-\mathrm{C}$ bond length hereafter. $\mathrm{C}-\mathrm{C}$ bond of benzene molecule is calculated to be $1.41 \AA$ long in the ground state. Upon excitation to $\mathrm{S}_{1}$ state, $\mathrm{C}-\mathrm{C}$ bonds of benzene are symmetrically elongated to a length $1.45 \AA$. On the other hand, asymmetric bond deformation is observed in $\mathrm{T}_{1}$ state; a pair of facing $\mathrm{C}-\mathrm{C}$ bonds get shortened to about $1.37 \AA$, while the other four $\mathrm{C}-\mathrm{C}$ bonds are elongated to $c a$. $1.48 \AA$. The dissimilarity in geometry can be understood with the aid of natural transition orbital analysis of excited states; see Figure 2. A transition to a given excited state introduces additional nodes in the particle wavefunctions leading to longer bonds. As clearly seen, $\mathrm{S}_{1}$ state can be described by the two pairs of NTO wavefunctions with nearly equal weights. Since these two NTO pairs are complementary to each other such that asymmetric deformation induced by one combination can be fully compensated by the other. As a result, benzene has all equal C-C bonds in $\mathrm{S}_{1}$ state. On the other hand, $T_{1}$ state is characterized by the single dominant pair of hole-particle NTOs giving rise to asymmetric $\mathrm{C}-\mathrm{C}$ bond deformation. The difference in natures of singlet and triplet excited states likely stems from exchange energy; ${ }^{33}$ as singlet [triplet] state is destabilized
Table 1. Calculated geometric parameters $(\AA)$ and electronic transition energies $(\mathrm{eV})$ of benzene in the ground and excited states ${ }^{a}$

\begin{tabular}{cccc}
\hline & $\mathrm{S}_{0}$ & $\mathrm{~S}_{1}$ & $\mathrm{~T}_{1}$ \\
\hline $\mathrm{R}(\mathrm{C}-\mathrm{C})$ & 1.41 & 1.45 & $1.37 / 1.48$ \\
$\mathrm{R}(\mathrm{C}-\mathrm{H})$ & 1.10 & 1.09 & 1.09 \\
$\Delta \mathrm{E}_{\mathrm{VA}}{ }^{b}$ & & 5.09 & 4.17 \\
$\Delta \mathrm{E}_{\mathrm{VE}}{ }^{b}$ & & 4.73 & 3.35 \\
$\Delta \mathrm{E}_{\mathrm{ad} .}{ }^{b}$ & & 4.91 & 3.87 \\
Expt. & & $4.78^{c}, 4.79^{d}$ & $3.67^{b}$ \\
\hline
\end{tabular}

${ }^{a}$ All the values are derived at SOS-CIS( $\left.\mathrm{D}_{0}\right) /$ aug-cc-pVDZ level. ${ }^{b} \Delta \mathrm{E}_{\mathrm{VA}}$. and $\Delta \mathrm{E}_{\mathrm{VE}}$. correspond to vertical transition energies at ground-state and excite-state geometries, respectively: $\Delta \mathrm{E}_{\text {ad. }}$ represents adiabatic transition energy obtained via $\triangle \mathrm{SCF}$ method based upon optimized geometries in respective states. Refer the text for more details. ${ }^{c}$ Ref. $34 .{ }^{d}$ Ref. 35
$\mathrm{S}_{1}$
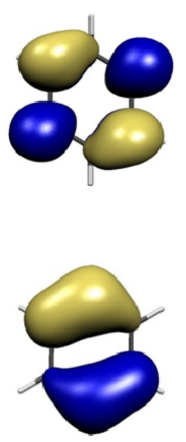

0.4990
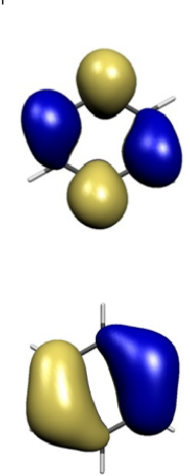

0.4989
$\mathrm{T}_{1}$
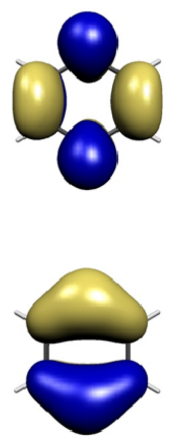

0.9291
Figure 2. Hole (bottom) and particle (top) wavefunctions of natural transition orbital for $S_{1}$ and $T_{1}$ states of benzene molecule. The values on the bottom correspond to the square of singular value of one-particle transition density matrix of $\operatorname{CIS}\left(\mathrm{D}_{0}\right), \lambda$.

[stabilized] by exchange energy, in singlet [triplet] states a molecular system would take the combination of small [large] exchange energy by minimizing [maximizing] the hole-particle wavefunction overlap. Indeed, the largest amplitude of hole wavefunction is located where the smallest amplitude of particle wavefunction is placed.

In terms of electronic transition, computation values are in fair agreement with experimental ones; for example, $\mathrm{S}_{1}$ is experimentally observed to be $c a .4 .78 \mathrm{eV}$ higher in energy than ground state $^{34,35}$ and the adiabatic energy difference between $S_{0}$ and $S_{1}$ states is calculated to be $c a$. $4.91 \mathrm{eV}$ in this study. Likewise, the first vibrational level of $\mathrm{T}_{1}$ state is reportedly $c a .3 .67 \mathrm{eV}$ above the ground state ${ }^{34}$ which is compatible with computed adiabatic transition energy of $c a$. $3.87 \mathrm{eV}$. We also note that the calculated Stokes shift for $\mathrm{S}_{1}$ state is relatively smaller than that for $T_{1}$ state $(0.36 \mathrm{eV} v s$ $0.82 \mathrm{eV}$ ), being consistent with more significant geometric relaxation in $\mathrm{T}_{1}$ state. Armed with this information, we now turn to excited dimers in the following section.

Dimer: T-shaped vs Face-to-Face Eclipsed Configurations. Figure 3 presents the computed potential energy curves (PEC) of benzene dimer. Note that geometry relaxation is 

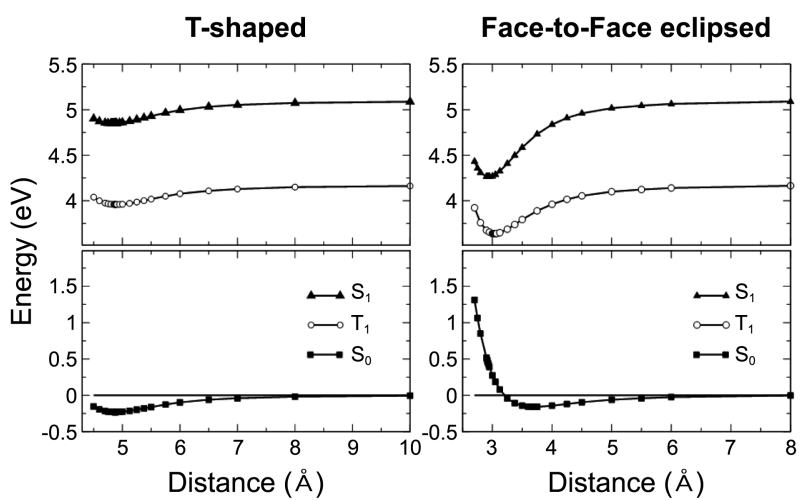

Figure 3. Potential energy curves of representative benzene dimers for the ground $\left(\mathrm{S}_{0}\right)$ and lowest lying excited $\left(\mathrm{S}_{1}\right.$ and $\left.\mathrm{T}_{1}\right)$ states as a function of intermolecular spacing.

not taken into account in this computation, and hence PEC is described as a function of distance between centers of mass of each molecule. Table 2 collects geometric parameters and binding energies of benzene dimers for the ground and excited states. We found BSSE to be such large that, with both BSSE and geometry relaxation taken into account, F2F-eclipsed dimer might be either very weakly bound or even unbound in $T_{1}$ state. In this report, however, we put more emphasis on the geometry relaxation effect on and spin-state dependence of excimer formation. Hence, our discussion will be based upon the CP-uncorrected energies.

In the case of T-shaped configuration, PECs for the ground and excited states evolve in a parallel way and therefore Equilibrium intermolecular distances, $R_{e}$, and binding energies, $-\Delta E$, are nearly the same; $R_{e}$ for $\mathrm{S}_{0}, \mathrm{~T}_{1}$, and $\mathrm{S}_{1}$ states are calculated to be $4.87,4.87$, and $4.82 \AA$, respectively, and $-\Delta E$ for these states range from $c a$. 0.22 to $0.24 \mathrm{eV}$. Presumably, in this configuration, the electronic transitions of one molecule are hardly affected by the other and intermolecular interactions in the excited states are dominated by those in the ground state.

In the case of F2F-eclipsed configuration, on the other hand, intermolecular interactions in the excited state are significantly different than those of the ground-state dimer. Our results state that intermolecular distances are 0.69-0.78 $\AA$ shorter in the excited states than that in the ground state when molecular structures are kept frozen, indicating that intermolecular interactions are stronger in the excited states.

Geometry relaxation does not change this picture dramatically; on the contrary, it renders this picture clearer. In the case of T-shaped dimer, two molecules in the ground state is calculated to be separated by a distance of $c a .4 .87 \AA$ for $\mathrm{S}_{0}$ state and those in the excited states are negligibly moved apart (the separation of $c a .4 .88 \AA$ ). Likewise, binding energies, $-\Delta E$, remain unaltered, i.e., ca. $0.23 \mathrm{eV}$ for the ground state $v s c a .0 .22 \mathrm{eV}$ for both excited states. Furthermore, geometry relaxation in the excited state occurs only in one of molecules. For instance, in $\mathrm{S}_{1}$ state, $\mathrm{C}-\mathrm{C}$ bonds of the molecule on the bottom in Figure 1 extend to a length $1.45 \AA$ but those of the other molecule are not affected. Indeed, both
Table 2. Optimal intermolecular distances $\left(\mathrm{R}_{\mathrm{e}}\right)$ and binding energies $(-\Delta \mathrm{E})$ of benzene dimers for the ground and the lowest-lying excite states

\begin{tabular}{|c|c|c|c|c|}
\hline & \multicolumn{2}{|c|}{ T-shaped } & \multicolumn{2}{|c|}{ F2F-eclipsed } \\
\hline & Frozen & Relaxed & Frozen & Relaxed \\
\hline \multicolumn{5}{|c|}{$\mathrm{R}_{\mathrm{e}}(\AA)$} \\
\hline $\mathrm{S}_{0}$ & 4.87 & 4.87 & 3.71 & 3.69 \\
\hline $\mathrm{T}_{1}$ & 4.87 & 4.88 & 3.03 & 2.94 \\
\hline $\mathrm{S}_{1}$ & 4.82 & 4.88 & 2.93 & 2.93 \\
\hline Expt. & & & \multicolumn{2}{|c|}{$3 \cdot 0-3.6^{b}$} \\
\hline \multicolumn{5}{|c|}{$-\Delta \mathrm{E}\left[-\Delta \mathrm{E}^{\mathrm{cp}}\right]^{a}(\mathrm{eV})$} \\
\hline $\mathrm{S}_{0}$ & $0.23[0.06]$ & $0.23[0.06]$ & $0.16[0.02]$ & $0.16[0.02]$ \\
\hline $\mathrm{T}_{1}$ & $0.22[0.05]$ & $0.22[0.05]$ & $0.53[0.20]$ & $\begin{array}{c}0.37 \\
{[-0.01]}\end{array}$ \\
\hline $\mathrm{S}_{1}$ & $0.24[0.06]$ & $0.22[0.05]$ & $0.83[0.45]$ & $0.74[0.35]$ \\
\hline Expt. & & & \multicolumn{2}{|c|}{$0.34,^{c}>0.36^{d}$} \\
\hline
\end{tabular}

${ }^{a}-\Delta \mathrm{E}$ and $-\Delta \mathrm{E}^{\mathrm{cp}}$ denote $\mathrm{CP}$-uncorrected and $\mathrm{CP}$-corrected binding energies, respectively. ${ }^{b, c, d}$ Experimental values correspond to singlet excimer. ${ }^{b}$ Ref. $18 .{ }^{c}$ Ref. $16 .{ }^{d}$ Ref. 17
$\mathrm{S}_{1}$
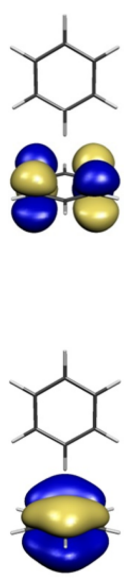

0.5084
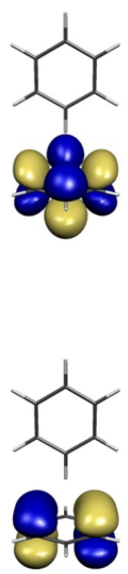

0.4886
$\mathrm{T}_{1}$
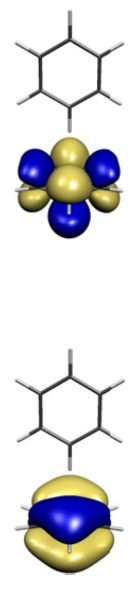

0.9393
Figure 4. Natural transition orbital pairs of T-shaped benzene dimer for $S_{1}$ and $T_{1}$ states: hole and particle wavefunctions are placed in the middle and on top, respectively; square of singular values of one-particle transition density matrix, $\lambda$, for each pair of NTOs are on the bottom.

for $\mathrm{S}_{1}$ and $\mathrm{T}_{1}$ state, a pair of hole and particle NTOs clearly show the local excitation in this configuration; see Figure 4. All these results add up to the conclusion that even if electron density distribution of the molecule that undergoes electronic transition would change, the impact would be marginal for this configuration and intermolecular interactions are dominated by those in the ground state; hence total interactions remain constant.

With regard to F2F-eclipsed dimer, geometry relaxation affects equilibrium distance, $R_{e}$, only marginally but leads to notable change in binding energy in the excited state; it reduces from $c a$. $0.53 \mathrm{eV}$ to $c a .0 .37 \mathrm{eV}$ for $\mathrm{T}_{1}$ state, and 
$\mathbf{S}_{1}$
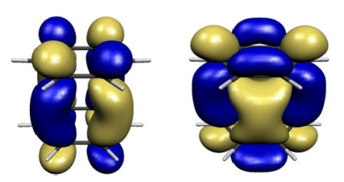

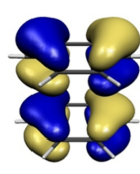

0.4651

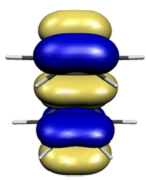

0.4650
$\mathbf{T}_{1}$
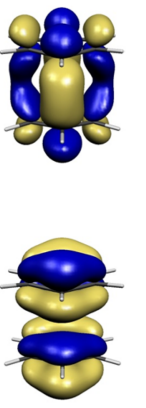

0.8580
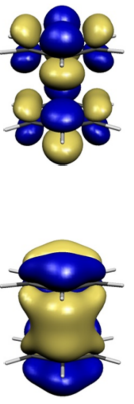

0.0753
Figure 5. Natural transition orbital pairs of F2F-eclipsed benzene dimer for $S_{1}$ and $T_{1}$ states: hole and particle wavefunctions are placed in the middle and on top, respectively; square of singular values of one-particle transition density matrix, $\lambda$, for each pair of NTOs are on the bottom.

changes from $c a .0 .83 \mathrm{eV}$ to $c a .0 .74 \mathrm{eV}$ in $\mathrm{S}_{1}$ state. NTO analyses of these states demonstrate that, unlike T-shaped configuration, hole-particle NTOs are totally delocalized over the dimer (Figure 5) and thus geometry relaxation effect is expected to be less than the monomer, giving rise to the decrease in the binding energy. Besides, as mentioned above, structural change was observed larger in $T_{1}$ state than $\mathrm{S}_{1}$ state and such is the reduction of binding strength upon geometry relaxation (ca. $0.16 \mathrm{eV} v s c a .0 .09 \mathrm{eV}$ for $\mathrm{T}_{1}$ and $\mathrm{S}_{1}$ states, respectively).

As noted from PECs, intermolecular interactions appear to be all different for each electronic state; molecules bind tighter in the excited state than the ground state. Previous results state that this is due to the strong molecular orbital interactions; as molecules come closer, molecular orbital interactions becomes stronger such that HOMO is destabilized while LUMO is stabilized, leading to significantly reduced HOMO-LUMO gap and hence reduced transition energy. ${ }^{25,26}$ It is also found to be intriguing that molecules interact even stronger in $S_{1}$ state than $T_{1}$ state in this configuration. This might be ascribed, in part, to the fact that the localized nature of triplet wavefunction ${ }^{36,37}$ weakens to some extent the electronic coupling between two localized triplet states, i.e., state for the dimer with one molecule in $\mathrm{T}_{1}$ state and the other in the ground state and vice versa. A closer comparison of NTOs for $S_{1}$ and $T_{1}$ states, however, suggests that the different behavior of exchange energy for the singlet and triplet states might be in play. For singlet excimer, hole wavefunction corresponds to the orbitals of anti-bonding type combination of HOMOs of benzene molecule and particle wavefunction points to those of bonding-type combination of their LUMOs. The exchange energy of these delocalized wavefunction is expected to decrease due to the increased average distance between hole and electron and thus further stabilize singlet state. In the same sense, despite the significantly small band-gap, the transition between the delocalized orbitals somewhat destabilizes triplet state in terms of exchange energy. Besides, the larger-band-gap tran- sition between anti-bonding-type hole NTO and bondingtype particle NTO also contributes to $\mathrm{T}_{1}$ excimer state by $c a$. 7.5\% (Figure 5). This might be the case that the enhancement of exchange energy leads to the reduction of excimer binding energy for $T_{1}$ state. To draw a more definite picture, further work is warranted and is on the way.

\section{Conclusion}

By means of $a b$ initio theoretical calculations, we studied the effect of geometry relaxation on and spin-dependence of the formation of benzene excimer. We uncovered that potential energy curve calculations of dimer with the frozen monomer structure can be effective to estimate the equilibrium distance. In addition, in the case of T-shaped configuration in which intermolecular interactions in the excited states are dominated by those in the ground state, geometry relaxation effects remain negligible. Without geometry relaxation, however, the overestimation of excimer binding energy was inevitable for F2F-eclipsed dimer where wavefunctions for the excited state tend to be delocalized. In this configuration, we also found that the strength of interactions depends upon the spin-state of excimer in which exchange energy plays an important role. Given that the spin-statistics, in particular, the energy difference between singlet and triplet excited states is considered to be one of key factors in modern organic electronics, we believe that our results provide an important inkling to pave a new viable way of manipulating it.

Acknowledgments. The author would like to thank Prof. Y. M. Rhee for fruitful discussion. This work was supported by Kyonggi University Research Grant 2010-2011.

\section{References}

1. Somerharju, P. Chem. Phys. Lipid 2002, 116, 57.

2. Conlon, P.; Yang, C. J.; Wu, Y.; Chen, Y.; Martinez, K.; Kim, Y.; Stevens, N.; Marti, A. A.; Jockusch, S.; Turro, N. J.; Tan, W. J. Am. Chem. Soc. 2007, 130, 336.

3. Wu, C.; Wang, C.; Yan, L.; Yang, C. J. J. Biomed. Nanotech. 2009, 5, 495 .

4. Kim, H. G.; Lee, C.-W.; Yun, S.; Hong, B. H.; Kim, Y.-O.; Kim, D.; Ihm, H.; Lee, J. W.; Lee, E. C.; Tarakeshwar, P.; Park, S.-M.; Kim, K. S. Org. Lett. 2003, 5, 3971.

5. Lee, E. C.; Kim, D.; Jurečka, P.; Tarakeshwar, P.; Hobza, P.; Kim, K. S. J. Phys. Chem. A 2007, 111, 3446.

6. Günes, S.; Neugebauer, H.; Sariciftci, N. S. Chem. Rev. 2007, 107.

7. Shirota, Y.; Kageyama, H. Chem. Rev. 2007, 107, 953.

8. D'Andrade, B.; Forrest, S. R. Chem. Phys. 2003, 286.

9. Kim, D.; Brédas, J.-L. J. Am. Chem. Soc. 2009, 131, 11371.

10. Williams, E. L.; Haavisto, K.; Li, J.; Jabbour, G. E. Advanced Materials 2007, 19.

11. Jankus, V.; Monkman, A. P. Adv. Funct. Mater. 2011, 21, 3350.

12. Brédas, J.-L.; Beljonne, D.; Coropceanu, V.; Cornil, J. Chem. Rev. 2004, 104, 4971.

13. Smith, M. B.; Michl, J. Chem. Rev. 2010, 110, 6891.

14. Clarke, T. M.; Durrant, J. R. Chem. Rev. 2010, 110, 6736.

15. Lee, E. C.; Hong, B. H.; Lee, J. Y.; Kim, J. C.; Kim, D.; Kim, Y.; Tarakeshwar, P.; Kim, K. S. J. Am. Chem. Soc. 2005, 127, 4530.

16. Azumi, T.; McGlynn, S. P. J. Chem. Phys. 1964, 41, 3131. 
17. Hirayama, F.; Lipsky, S. J. Chem. Phys. 1969, 51, 1939.

18. Förster, T. Angew. Chem. Int. Ed. 1969, 8, 333.

19. Huenerbein, R.; Grimme, S. Chem. Phys. 2008, 232, 362.

20. Olaso-González, G.; Roca-Sanjuán, D.; Serrano-Andrés, L.; Merchán, M. J. Chem. Phys. 2006, 125, 231102.

21. Amicangelo, J. C. J. Phys. Chem. A 2005, 109, 9174.

22. Velardez, G. F.; Lemke, H. T.; Breiby, D. W.; Nielsen, M. M.; Møller, K. B.; Henriksen, N. E. J. Phys. Chem. A 2008, 112, 8179.

23. Rocha-Rinza, T.; Christiansen, O. Chem. Phys. Lett. 2009, 482, 44.

24. Pabst, M.; Lunkenheimer, B.; Köhn, A. J. Phys. Chem. C 2011, 115,8335 .

25. Shirai, S.; Iwata, S.; Tani, T.; Inagaki, S. J. Phys. Chem. A 2011, $115,7687$.

26. Kołaski, M.; Arunkumar, C. R.; Kim, K. S. J. Chem. Theor. Compt. 2013, 9, 847.

27. Luo, Y.; Aziz, H. Adv. Funct. Mater. 2010, 20, 1285.

28. Zhang, Y.; Forrest, S. R. Phys. Rev. Lett. 2012, 108, 267404.

29. Grimme, S. J. Chem. Phys. 2003, 118, 9095.

30. Casanova, D.; Rhee, Y. M.; Head-Gordon, M. J. Chem. Phys. 2008, 128, 164106

31. Xantheas, S. S. J. Chem. Phys. 1996, 104, 8821.

32. Shao, Y.; Molnar, L. F.; Jung, Y.; Kussmann, J.; Ochsenfeld, C.;
Brown, S. T.; Gilbert, A. T. B.; Slipchenko, L. V.; Levchenko, S V.; O'Neill, D. P.; DiStasio, R. A., Jr.; Lochan, R. C.; Wang, T.; Beran, G. J. O.; Besley, N. A.; Herbert, J. M.; Yeh Lin, C.; Van Voorhis, T.; Hung Chien, S.; Sodt, A.; Steele, R. P.; Rassolov, V. A.; Maslen, P. E.; Korambath, P. P.; Adamson, R. D.; Austin, B.; Baker, J.; Byrd, E. F. C.; Dachsel, H.; Doerksen, R. J.; Dreuw, A.; Dunietz, B. D.; Dutoi, A. D.; Furlani, T. R.; Gwaltney, S. R.; Heyden, A.; Hirata, S.; Hsu, C.-P.; Kedziora, G.; Khalliulin, R. Z.; Klunzinger, P.; Lee, A. M.; Lee, M. S.; Liang, W.; Lotan, I.; Nair, N.; Peters, B.; Proynov, E. I.; Pieniazek, P. A.; Min Rhee, Y.; Ritchie, J.; Rosta, E.; David Sherrill, C.; Simmonett, A. C.; Subotnik, J. E.; Lee Woodcock Iii, H.; Zhang, W.; Bell, A. T.; Chakraborty, A. K.; Chipman, D. M.; Keil, F. J.; Warshel, A.; Hehre, W. J.; Schaefer Iii, H. F.; Kong, J.; Krylov, A. I.; Gill, P. M. W.; Head-Gordon, M. Phys. Chem. Chem. Phys. 2006, 8, 3172.

33. Ratner, M. A.; Schatz, G. C. Introduction to Quantum Mechanics in Chemistry; Pearson Education, Inc. (Prentice Hall): Upper Saddle River, NJ., 2001.

34. Doering, J. P. J. Chem. Phys. 1977, 67, 4065.

35. Hiraya, A.; Shobatake, K. J. Chem. Phys. 1991, 94, 7700.

36. Rioux, F. J. Chem. Educ. 2007, 84, 358.

37. Salman, S.; Kim, D.; Coropceanu, V.; Brédas, J.-L. Chem. Mater. 2011, 23, 5223. 